\title{
Charge-Coupled-Device and Charge-Injection-Device Theory and Application
}

\author{
James R. Janesick \\ Jet Propulsion Laboratory \\ California Institute of Technology \\ 4800 Oak Grove Drive \\ Pasadena, California 91109
}

This special issue of Optical Engineering is the third of three consecutive issues on charge-coupled devices. Papers contributed to this issue present various topics related to theoretical aspects of the $C C D$ and include three papers discussing a close cousin of the CCD, the charge-injection device (CID).

As with the other issues in this series, we also include here a few papers that describe specific CCD-based instruments and that illustrate the diversity of CCD applications. It becomes very apparent when reading the papers collected in these issues that the CCD has made its greatest contribution in the area of scientific visible imaging. The sensor has become one of the most commonly used detectors for ground-based astronomy. Only a dozen years have passed since the first two-dimensional imaging CCDs were fabricated, and today there is no major telescope operated in the world that does not have a CCD camera as part of its observing arsenal. At present, earthbased telescopes equipped with CCD cameras are being used to detect objects fainter than 26th magnitude, some 100 times fainter than the photographic limit of the Palomar 200-inch telescope. Larger optical telescopes now in the design phase (e.g., the 393-inch Keck telescope) plan to use several new Tektronix 2048×2048 CCDs (see paper by Blouke, Corrie, et al. in the September issue) at their focal planes to push the seeing limit even farther.

CCDs will also be incorporated in most upcoming NASA flight missions. For example, eight $800 \times 800 \mathrm{CCDs}$ will be used in the Wide Field/Planetary Camera (WF/PC) on the Hubble Space Telescope, a 90 -inch telescope ready for launch using the Space Shuttle to study the universe over a 10 -year period. The WF/PC instrument has the potential to see 10 times farther than most ground-based CCD cameras, thereby allowing astronomers to observe almost to the edge of the universe some $10^{23}$ miles away. Closer to home, the Galileo mission to Jupiter utilizes a single $800 \times 800$ CCD and will study in detail the Jovian system over a 5-year period. Other proposed NASA missions to the sun (HRSO), Mars (Mars Observer), Saturn (Cassini), and a comet and asteroid (CRAF) all plan to fly CCD cameras as part of their scientific payloads. Recently there has been a great deal of interest in using CCDs for imaging in the soft x-ray part of the electromagnetic spectrum. CCDs have been chosen as detectors for the Advanced $X$-ray Astrophysics Facility (AXAF), a future NASA mission to be launched a decade from now to study the universe in the $x$-ray (see papers by Luppino et al. and Doty et al. in this issue).

Ironically, the United States (inventor of the CCD) up to this point has not flown a scientific CCD imaging camera, although other countries have successfully done so (see paper by Seige and Ress in this issue). For example, ESA's Giotto, a European mission flown last year, produced outstanding CCD images of comet Halley's nucleus. CCD imagers were also used by the Russians and Japanese to study this famous comet. It is hoped that by the end of this decade one or more American-based instruments (e.g., WF/PC and Galileo) also will be operating in space.

Closer to home, CCDs now serve as detectors in a host of laboratory instruments (see papers by Naday et al. on "Detector with charge-coupled-device sensor for protein crystallography with synchrotron $x$ rays" and Yates et al. on "Characterization of electro-optic anomalies associated with transient response of fast readout charge-coupled devices," both in the August issue, and also the paper by Tincknell et al. on "Fast megapixel charge-coupleddevice image acquisition and analysis system for high energy nuclear physics" in this issue). The list of applications for CCDs in the laboratory is already extensive and continues to grow.

While CCDs enable the development of many exciting instruments, there remain impediments to their use. CCDs are still very expensive; a backside-illuminated $2048 \times 2048$ CCD presently lists for $\$ 80,000$, or 19 cents per pixel. Devices with standard TV resolution range from $\$ 2,000$ to $\$ 10,000$ (1.2 to 6 cents per pixel), depending on their performance and quality. Perhaps the most significant disadvantage of CCDs is the amount of ancillary electron- 
ics needed to make them work. Prime among these accessories is a powerful computer. CCD cameras generate enormous amounts of high quality data, often placing significant data processing demands on the user. For example, a single $2048 \times 2048$ CCD image holds the same information as a 7-million-word book (equivalent to about 6 encyclopedias). These data have to be stored, calibrated, and analyzed, a very expensive enterprise. Finally, a CCD instrument requires a support team that must include a variety of electronics and software talent. Nevertheless, because of their attributes, CCDs are attracting an everexpanding scientific user community, and this trend will surely continue as CCDs and computers become more economical to acquire and operate.

I would again like to express my thanks to the many authors and coauthors who have shared their CCD experiences in these special issues. I want to especially thank Jack Gaskill, Martha Stockton, Eric Pepper, and their staff for producing the issues you now read. I express my appreciation to Deborah Durham, who persevered in the receipt, review, revision, and acceptance of the 44 papers that appear in these issues. Finally, I want to thank Andy Collins, Chris Stevens, Bob Lockhart, and Kane Casani, my management staff here at JPL, for supporting this effort from beginning to end.

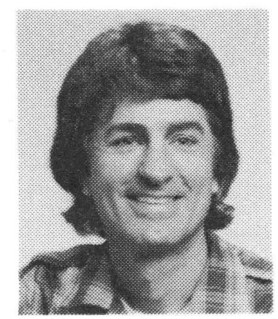

James R. Janesick was born in Orange, California, in 1947. He received his master's degree in electronic engineering from the University of California at Irvine, majoring in lasers and masers. Since January 1973 he has been working at the Jet Propulsion Laboratory/California Institute of Technology developing charge-coupled devices for the Wide Field/ Planetary Camera for the Hubble Space Telescope, Galileo Solid State Imaging Camera, and other NASA space imaging systems. He is author of 15 papers on the subject of CCDs, has contributed to several NASA tech briefs, and holds three patents on various CCD innovations. $\mathrm{He}$ received the Exceptional Engineering Achievement medal from NASA in 1982 and a NASA Achievement Award in 1986. He is currently involved in the development of narrow-band-gap monolithic CCD sensors for infrared and $\mathrm{x}$-ray imaging space instruments. 